Hannes Maxin*

\title{
Corporate Venture Capital im Bankensektor: Eine Fallstudie
}

\section{Zusammenfassung}

Die Digitalisierung der Gesellschaft beeinflusst zunehmend das klassische Bankgeschäft, was durch die Erfolge vieler junger FinTech-Unternehmen verdeutlicht wird. Der damit verbundene Innovationsdruck führte dazu, dass die Commerzbank AG im Oktober 2013 die Main Incubator GmbH gründete. Mithilfe dieser Corporate Venture Capital-Gesellschaft (CVC-Gesellschaft) versucht die Frankfurter Großbank, eine Kooperation mit den neuen Wettbewerbern einzugehen, um mögliche Synergiepotenziale für das eigene Unternehmen zu realisieren. In der Literatur werden CVC-Aktivitäten vornehmlich Großunternehmen aus dem Industrie- sowie Dienstleistungsbereich zugesprochen, die nicht dem Bankensektor angehören. Vor diesem Hintergrund stellt der Main Incubator einen neuartigen Fall dar, der im Rahmen einer Fallstudie analysiert werden soll, um charakteristische Merkmale für die Bankenbranche aufzuzeigen.

JEL-Classification: G24, M13.

Keywords: Corporate Venture Capital; Banken; Fallstudie; Prinzipal-Agent; FinTechUnternehmen.

Corporate venture capital; Banks; Case study; Principal-agent; FinTech firms.

\footnotetext{
* M.Sc. Hannes Maxin, Institut für Mikroökonomik, Leibniz Universität Hannover, Wissenschaftlicher Mitarbeiter, Königsworther Platz 1, 30167 Hannover, E-Mail: maxin @mik.uni-hannover.de. Der Verfasser dankt den Mitarbeitern der Main Incubator GmbH für ihre Unterstützung bei der Erstellung dieser Fallstudie sowie Frau Prof. Dr. Heidrun Hoppe-Wewetzer und den Teilnehmern des Brownbag Seminars derLeibniz Universität Hannover für ihre hilfreichen Anmerkungen.
} 


\section{Einleitung und Forschungsvorhaben}

Innovationen stehen im unmittelbaren Zusammenhang mit der Gewinnmaximierung von Unternehmen. Sie sind eine Grundlage dafür, mögliche Vorteile gegenüber der Konkurrenz zu schaffen, was sich in einer Steigerung der Erlöse beziehungsweise in einer Senkung der Kosten äußern kann. ${ }^{1}$ Entgegen der Schumpeter-Hypothese geht die ,innovative Kraft" dabei nicht ausschließlich von etablierten Großunternehmen aus. Insbesondere werden nur sehr wenige radikale Innovationen von diesen Organisationen realisiert. ${ }^{2}$ Solche einflussreichen Neuerungen finden vielmehr ihren Ursprung in neu gegründeten Wirtschaftseinheiten. ${ }^{3}$

Großunternehmen müssen sich gegenüber dieser Konkurrenz behaupten, wodurch ein zunehmender Innovationsdruck entsteht. ${ }^{4}$ Es bedarf folglich einer fortwährenden Anpassung des FuE-Prozesses sowie der Erschließung neuer Quellen für Innovationen. ${ }^{5}$ Eine Möglichkeit zur Umsetzung stellt CVC dar. Hierunter versteht man eine Beteiligung von Großunternehmen an jungen Wirtschaftseinheiten, um an deren Ideen zu partizipieren. Als Gegenleistung erhalten die jungen Organisationen neben Finanzkapital auch nichtmonetäre Unterstützung, womit anfangs fehlende Ressourcen für die Unternehmensentwicklung eine Bereitstellung erfahren. Die Großunternehmen müssen die Investitionen nicht zwangsweise selbst durchfuihren, sondern können auf spezielle Tochtergesellschaften zurückgreifen, die als CVC-Gesellschaften bezeichnet werden.

In Deutschland handelt es sich beim CVC um ein vergleichsweise neues Phänomen, das von der hiesigen Forschung bisher wenig berücksichtigt wurde. Nimmt man die Anzahl der existierenden CVC-Gesellschaften als Relevanzmaßstab für den Stellenwert des CVC aus Sicht der Großunternehmen, kann allerdings eine zunehmende Bedeutung verzeichnet werden. So gab es im Jahr 2005 nur 14 solcher Tochtergesellschaften, während im Jahr 2014 schon 37 existierten. $^{6}$

In der Literatur werden zu den Großunternehmen, denen CVC-Aktivitäten zugesprochen werden, insbesondere Industrie- sowie Dienstleistungsunternehmen gezählt, die nicht

\footnotetext{
${ }^{1}$ Vgl. Greenhalgh/Rogers (2010), S. 119 ff.

2 Vgl. Stringer (2000), S. 71. Vgl. auch Christensen (1997), S. 125 f.

${ }^{3}$ Vgl. Utterback (1994), S. 209.

${ }^{4}$ Vgl. Kreysel (2006), S. 2.

5 Vgl. Chesbrough (2006), S. 51 ff. Vgl. auch Lerner (2010), S. 11.

${ }^{6}$ Laut Angabe des Bundesverbandes Deutscher Kapitalbeteiligungsgesellschaften.
} 
dem Bankensektor angehören. Dies ist im Wesentlichen darauf zurückführen, dass Banken durch die Finanzierung junger Unternehmen vor allem Kapitalgewinne anstreben und weniger innovative Vorteile verfolgen. ${ }^{7}$ Häufig fehlen den Banken allerdings auch die entsprechenden Fähigkeiten, um eine Risikoeinschätzung der jungen Wirtschaftseinheiten durchzuführen, wodurch vollends auf solche Investitionen verzichtet wird. Seit dem Jahr 2014 existieren in Deutschland allerdings zwei Unternehmen, die als Gegenbeispiele für diese Annahme betrachtet werden können: Der Main Incubator und CommerzVentures. Beide CVC-Gesellschaften gehören dem Commerzbank Konzern an und sind zurzeit als einzige aktive CVC-Gesellschaften in Deutschland anzusehen, die von einer Großbank gegründet wurden. ${ }^{8}$ Im Rahmen dieser Fallstudie soll der Main Incubator genauer untersucht werden, um mögliche Unterschiede $\mathrm{zu}$ den bestehenden Annahmen des CVC aufzuzeigen.

Zunächst soll im Rahmen von Kapitel 2 eine Einführung stattfinden, welche die Bedeutung und die Ziele des CVC umfasst. Darüber hinaus wird die Vorgehensweise von CVCGesellschaften betrachtet, wobei mögliche theoretische Probleme aus Sicht des PrinzipalAgent-Ansatzes berücksichtigt werden. In Kapitel 3 wird die eigentliche Fallstudie über den Main Incubator aufgeführt. Die empirischen Befunde werden in Kapitel 4 analysiert, um mögliche Besonderheiten des CVC im Bankenbereich aufzuzeigen. Kapitel 5 beschließt diesen Artikel mit einem Fazit.

\section{Innovationsförderung durch Corporate Venture Capital}

Innovationen können in zwei Arten unterschieden werden: Interne und externe Innovationen. ${ }^{9}$ Die erste Form beruht auf Erfindungen, die innerhalb eines Unternehmens entstehen, während die zweite Art Forschungsleistungen impliziert, welche außerhalb einer Organisation geschaffen werden. Dabei übernimmt eine Wirtschaftseinheit das erreichte Stadium einer extern entstandenen Idee und entwickelt diese weiter. ${ }^{10}$ Für Großunternehmen stellt CVC vor allem eine Möglichkeit dar, solche externen Innovationen zu generieren. Dahingehend wird diesem Artikel eine Hypothese zugrunde gelegt, die als wesentliche Begründungen für den Einsatz von CVC anzusehen ist: Zwischen Großunterne hmen

\footnotetext{
${ }^{7}$ Vgl. Kreysel (2006), S. 37. Vgl. auch Hardenberg (1989), S. 79.

${ }^{8}$ Laut Angabe des Bundesverbandes Deutscher Kapitalbeteiligungsgesellschaften.

9 Vgl. Witt (2005), S. 260.

10 Vgl. Albach (1994), S. 64.
} 
und jungen Unternehmen bestehen komplementäre Innovationspotenziale, die Synergie n als sinnvolle Option offerieren. ${ }^{11}$

Junge Unternehmen können genau mit den Eigenschaften aufwarten, die bei etablierten Wirtschaftseinheiten zumeist fehlen. Dynamische Organisationsstrukturen und die damit verbundenen Freiräume fördern die Entstehung von neuem Wissen und ermöglichen Innovationen. ${ }^{12}$ Ein grundlegendes Problem von jungen Unternehmen im Gegensatz zu den Großunternehmen ist allerdings die unzureichende Ressourcenausstattung. Insbesondere das Finanzkapital kann als zentrale Entwicklungskomponente von neu gegründeten Wirtschaftseinheiten bezeichnet werden. ${ }^{13}$

\subsection{Definition und Ziele des Corporate Venture Capital}

Für eine ausreichende finanzielle Basis genügen den jungen Unternehmen bis zur StartupPhase häufig die eigenen monetären Mittel und öffentliche Fördermittel. Dies ist spätestens ab der First Stage nicht mehr gegeben, sodass eine finanzielle Lücke entstehen kann. Aufgrund hoher Investitionsrisiken und Imperfektionen auf dem Finanzkapitalmarkt stellt Fremdfinanzierung zunächst keine relevante Option dar. In gleicher Weise ist eine Erhöhung des Eigenkapitals über die Wertpapierbörsen nicht möglich. Die bestehende monetäre Lücke wird vielmehr durch das sogenannte Venture Capital (VC) geschlossen. ${ }^{14}$ Erfolgt die Bereitstellung des VC dabei durch ein Großunternehmen, ist die Bezeichnung CVC zu verwenden. Die im Folgenden aufgezeigten Merkmale einer CVCFinanzierung bilden die Grundlage dieses Artikels:

- CVC umfasst Finanzkapital, welches jungen Unternehmen in Form von Minderheitsbeteiligungen überlassen wird. Großunternehmen, die sich an jungen Wirtschaftseinheiten beteiligen, werden in der Folge auch als Mutterunternehmen bezeichnet. $^{15}$

- Bei den jungen Unternehmen handelt es sich vorzugweise um Organisationen, die sich in der Seed-, Start-up- oder First-Stage-Phase befinden. Nach erfolgter Beteiligung kann für die junge Wirtschaftseinheit ebenfalls der Begriff Portfolio unternehmen beziehungsweise Venture verwendet werden. ${ }^{16}$

\footnotetext{
${ }^{11} \mathrm{Vgl}$. Schween (1996), S. 64 f.

12 Vgl. Schween (1996), S. 74 m.w.N.

13 Vgl. Kreysel (2006), S. 23, 27.

${ }^{14}$ Vgl. Schefczyk (1998), S. 36 f.; Lerner/Tåg (2013), S. 155 m.w.N.

${ }^{15}$ Vgl. Schween (1996), S. 21

${ }^{16} \mathrm{Vgl}$. Haid (2004), S. 265.
} 
- Neben dem Finanzkapital erfahren die Ventures auf direkte und indirekte Weise eine nicht-monetäre Hilfe. Für die Unterstïtzung räumen die Portfoliounternehmen dem Mutterunternehmen zumeist Informations-, Kontroll- und Mitspracherechte ein. ${ }^{17}$

- Als Ergebnis der CVC-Aktivität kann ein Kapitalgewinn durch den Verkauf von Portfoliounternehmen-Anteile entstehen. Primär versucht das Mutterunternehmen allerdings strategische Vorteile zu realisieren. ${ }^{18}$ Diese duale Zielverfolgung stellt eine klare Abgrenzung zum Vorgehen klassischer VC-Gesellschaften dar, welche vornehmlich die Generierung von Kapitalgewinnen anstreben. ${ }^{19}$

Die strategischen Vorteile verdeutlichen dabei den positiven Effekt, der oben beschriebenen komplementären Innovationspotenziale für die Großunternehmen. Durch die synergetische Zusammenarbeit von einem Mutterunternehmen und seinem Venture kann somit eine „Win-Win-Situation“ entstehen. ${ }^{20}$ Während das junge Unternehmen eine monetäre und nicht-monetäre Unterstützung erhält, kann das Großunternehmen verschiedene strategische Ziele realisieren. Dazu zählen beispielsweise die Technologiebeobachtung (Window on Technology), der Transfer von Produkt- und Prozesswissen, die Übertragung der innovativen Kultur sowie die Sicherung der Absatz- und Beschaffungsmärkte. ${ }^{21}$ Dabei gilt es zu beachten, dass die Geschäftsmodelle der Ventures auch mit der Branche des Mutterunternehmens kompatibel sind, damit die Synergiepotenziale realisiert werden können. ${ }^{22}$

\subsection{Formen von Corporate Venture Capital}

Das Mutterunternehmen kann seine Investition auf drei verschiedene Arten durchführen: Als direktes, indirektes oder internes CVC. Abbildung 1 gibt einen Überblick über diese Methoden:23

\footnotetext{
17 Vgl. Lorenz/Seeliger (2000), S. 658.

18 Vgl. Sykes (1990), S. 38.

19 Vgl. Schween (1996), S. 21.

20 Vgl. Lorenz/Seeliger (2000), S. 658

${ }^{21}$ Vgl. Witt (2005), S. 263 f; Schween (1996), S. 85.

22 Vgl. Gompers/Lerner (2000), S. 28.

${ }^{23}$ CVC kann in weitere Formen differenziert werden. Vereinfachend werden nur drei Arten aufgeführt.
} 
Abbildung 1: Formen der CVC-Finanzierung.

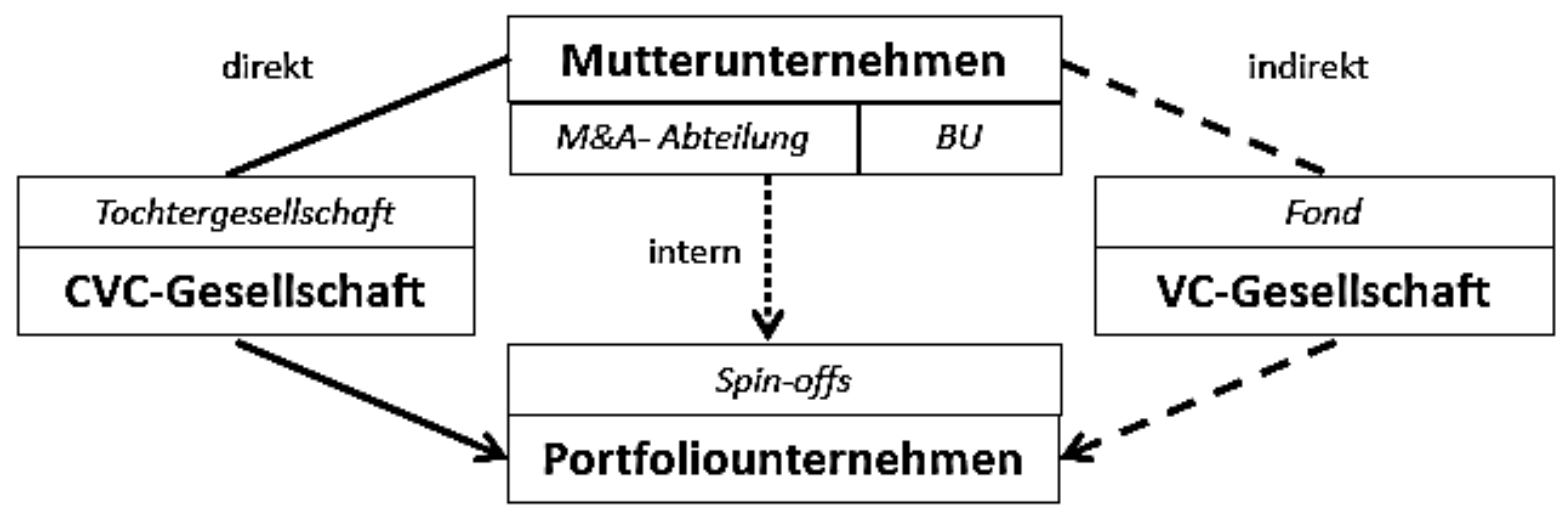

Bei der indirekten Form finanzieren Fonds, welche von VC-Gesellschaften geführt werden, die jungen Unternehmen. ${ }^{24}$ Oftmals wird eine solche Vorgehensweise als Einstieg in den CVC-Bereich gewählt, wenn das Mutterunternehmen noch keine Erfahrungen gesammelt hat. ${ }^{25}$ Allerdings können durch den Einsatz eines externen Finanzintermediäres nur unzureichend strategische Vorteile erzielt werden. ${ }^{26}$

Führen Mergers \& Acquisitions-Abteilungen oder Business Units die Investitionen durch, wird der Begriff ,internes CVC“beziehungsweise „Corporate Venturing“ verwendet. Dabei agieren die Abteilungen nicht aktiv auf dem CVC-Markt, wodurch sie häufig von den jungen Unternehmen unbeachtet bleiben. Als Zielgruppe gelten vor allem unternehmenseigene Spin-offs. ${ }^{27}$

Direktes CVC wird durch eine rechtlich selbstständige Tochtergesellschaft realisiert. Eine solche CVC-Gesellschaft agiert eigenverantwortlich am Markt, wobei zumeist der Unternehmensname einen Hinweis auf das entsprechende Großunternehmen gibt. ${ }^{28}$ Im Gegensatz zum indirekten CVC kann den strategischen Zielen bei der direkten Form eher entsprochen werden, da eine engere Zusammenarbeit zwischen Großunternehmen und Portfoliounternehmen besteht. ${ }^{29}$ Aufgrund des eingangs formulierten Forschungsvorhabens soll das direkte CVC als Grundlage dieses Artikels angesehen werden.

\footnotetext{
${ }^{24}$ Vgl. Lorenz/Seeliger (2000), S. 659.

25 Vgl. Neubecker (2006), S. 100.

26 Vgl. McNally (1997), S. 43 f. m.w.N.

27 Vgl. Lorenz/Seeliger (2000), S. 659.

28 Vgl. Lorenz/Seeliger (2000), S. 659.

29 Vgl. McNally (1997), S. 123 f.
} 
Grundsätzlich fungiert die CVC-Gesellschaft als Intermediär zwischen Großunternehmen und Venture. Hierbei gilt es, beiden Parteien gerecht zu werden und die jeweiligen Interessen trotz möglicher Zielkonflikte zu berücksichtigen. ${ }^{30}$ Eine Anwendung des Prinzipal-Agent-Ansatzes zeigt jedoch, dass aus theoretischer Sicht eine zweistufige PrinzipalAgent-Beziehung vorliegt. Die erste Ebene besteht aus dem Mutterunternehmen (Prinzipal) $^{31}$ und der CVC-Gesellschaft (Agent), während die zweite Stufe die CVC-Gesellschaft (Prinzipal) und das Portfoliounternehmen (Agent) umfasst. Aufgrund von Informationsasymmetrien kann es auf beiden Stufen zu erheblichen Interessenskonflikten kommen. ${ }^{32}$

Hierbei verdeutlicht die zweite Stufe, die eingangs beschriebenen Imperfektionen auf dem Finanzkapitalmarkt. Es kann demnach bei der Finanzierung von jungen Unternehmen zur Adversen Selektion sowie zum Moral Hazard kommen. ${ }^{33}$ In gleicher Weise wie eine reine VC-Gesellschaft verfugt eine CVC-Gesellschaft allerdings über Lösungsansätze, die zur Überwindung dieser Schwierigkeiten beitragen. Die entsprechenden Möglichkeiten werden in den Abschnitten 2.3 und 2.4 genauer betrachtet.

\subsection{Der Auswahlprozess von Corporate Venture Capital-Gesellschaften}

Die Auswahl geeigneter Portfoliounternehmen beginnt mit der Identifizierung von potentiellen Beteiligungsprojekten und der Kontaktaufnahme, was als Deal Flow bezeichnet wird. ${ }^{34}$ Zur Anbahnung dieses „Stroms“ kann die CVC-Gesellschaft entweder eine aktive oder eine passive Vorgehensweise verfolgen. Bei der ersten Option werden gezielt Marketingmaßnahmen eingesetzt. ${ }^{35}$ Von einer passiven Vorgehensweise wird gesprochen, wenn die Initiative vom jungen Unternehmen selbst oder Dritten ausgeht. ${ }^{36}$

Die Einreichung der Geschäftsidee erfolgt zumeist mithilfe eines Businessplans, woraufhin eine zweistufige Beteiligungswürdigkeitsprüfung einsetzt. Dem Prinzipal-Agent-Ansatz folgend, ist dieses Screening ein Ergebnis des unvollkommenen Finanzkapitalmarktes. Für die CVC-Gesellschaft (Prinzipal) liegt die Schwierigkeit vor, die Qualität des

\footnotetext{
30 Vgl. Chesbrough/Socolof(2000), S. 15 f.

${ }^{31} \mathrm{Im}$ Rahmen dieses Artikels wird bei Anwendung der Prinzipal-Agent-Theorie jeweils in Klammern stehen, welcher Akteur als Agent beziehungsweise Prinzipal anzusehen ist.

32 Vgl. Witt (2005), S. 265 f. m.w.N.

${ }^{33}$ Vgl. Lerner/Tåg (2013), S. 155 m.w.N; Schween (1996), S. 137 ff.

34 Vgl. Kreysel (2006), S. 72; Zemke (1995), S. 28.

35 Vgl. Schröder (1992), S. 159 f.

${ }^{36}$ Vgl. Neubecker (2006), S. 85; Kollmann (2003), S. 324.
} 
potentiellen Ventures (Agent) zu beurteilen (Hidden Characteristics). ${ }^{37}$ Demnach kann das Problem der adversen Selektion auftreten. Gute Bewerber werden möglicherweise von der Tochtergesellschaft aufgrund unzureichender Informationen unterbewertet, wodurch eine Negativselektion einsetzen kann. ${ }^{38}$ Der Prinzipal muss somit versuchen, die Informationsdefizite und die damit verbundenen Unsicherheiten zu reduzieren.

Zunächst erfolgt ein Initial Screening durch die Mitarbeiter der CVC-Gesellschaft. ${ }^{39}$ Siegel/Siegel/MacMillan haben fünf Kriteriengruppen definiert, anhand derer das Geschäftskonzept zumeist untersucht wird: Die Persönlichkeit sowie die Erfahrung der Gründer, die Produkteigenschaften, die Marktgegebenheiten und die finanziellen Aussichten. ${ }^{40}$ Dahingehend kann auch der Businessplan betrachtet werden, dem neben seiner Funktion als umfassende Informationsquelle zudem als Beurteilungskriterium eine immense Bedeutung zugesprochen wird. ${ }^{41}$ Zwar stellt ein gut geschriebener Businessplan keine Gewährleistung für eine erfolgreiche Entwicklung des Portfoliounternehmens dar, dennoch kann er als wichtiger Indikator für die Gründerteamqualität angesehen werden. ${ }^{42}$

Nach dem Initial Screening werden ungefähr 25 Prozent der ursprünglichen Bewerber in einem zweiten Schritt genauer analysiert, was als sogenannte Due Diligence bezeichnet wird. ${ }^{43}$ In dieser Phase rückt nunmehr das Wissen interner Experten des Mutterunternehmens sowie externer Spezialisten in den Vordergrund. Es werden insbesondere betriebswirtschaftliche, rechtliche und technische Merkmale des Bewerbers intensiv untersucht. ${ }^{44}$

Im Anschluss an das Auswahlwahlverfahren finden die Beteiligungs verhandlungen statt. Hierbei werden die Konditionen der zukünftigen Partnerschaft festgelegt, was beispielsweise die Höhe der Beteiligungsfinanzierung, die Unternehmensbewertung sowie die Informations-, Kontroll- und Mitspracherechte umfasst. Besteht ein beidseitiges Einverständnis, kann der endgültige Vertrag zwischen beiden Parteien geschlossen werden. ${ }^{45}$ Insgesamt führen jedoch nur etwa drei Prozent aller eingegangenen Geschäftsideen am Ende zur Aufnahme eines Bewerbers ins Portfolio. ${ }^{46}$

\footnotetext{
37 Vgl. Heister (2010), S. 109 f.; Schultz (2011), S. 94, 172 f.

${ }^{38}$ Vgl. Schween (1996), S. 146; Amit/Glosten/Muller (1990), S. 107 ff.

39 Vgl. Schween (1996), S. 106.

${ }^{40}$ Vgl. Siegel/Siegel/MacMillan (1988), S. 237.

${ }^{41}$ Vgl. Gumpert (1994), S. 123; Neubecker (2006), S. 89.

${ }^{42}$ Vgl. Schultz (2011), S. 177.

43 Vgl. Fendel (1987), S. 160; Schefczyk (1998), S. 40.

${ }^{44}$ Vgl. Schultz (2011), S. 174 f.; Meier/Rasmussen-Bonne (2000), S. 204.

${ }^{45}$ Vgl. Poser (2003), S. 145.

46 Vgl. Schefczyk (1998), S. 40.
} 


\subsection{Die Corporate Venture Capital-Unterstützungsleistungen}

Im Anschluss an die Beteiligungsverhandlungen beginnt die operative Zusammenarbe it mit den Ventures. In diesem Zeitabschnitt erfahren die Portfoliounternehmen über die rein monetäre Komponente hinausgehende, umfangreiche Unterstützung. Die CVC-Gesellschaft versucht, die Unternehmensentwicklung nachhaltig positiv zu beeinflussen, um das eigene Investitionsrisiko zu reduzieren und den Wert des jungen Unternehmens zu steigern. ${ }^{47}$ Abbildung 2 verdeutlicht die verschiedenen Komponenten:

Abbildung 2: Leistungen der CVC-Unterstützungsphase.

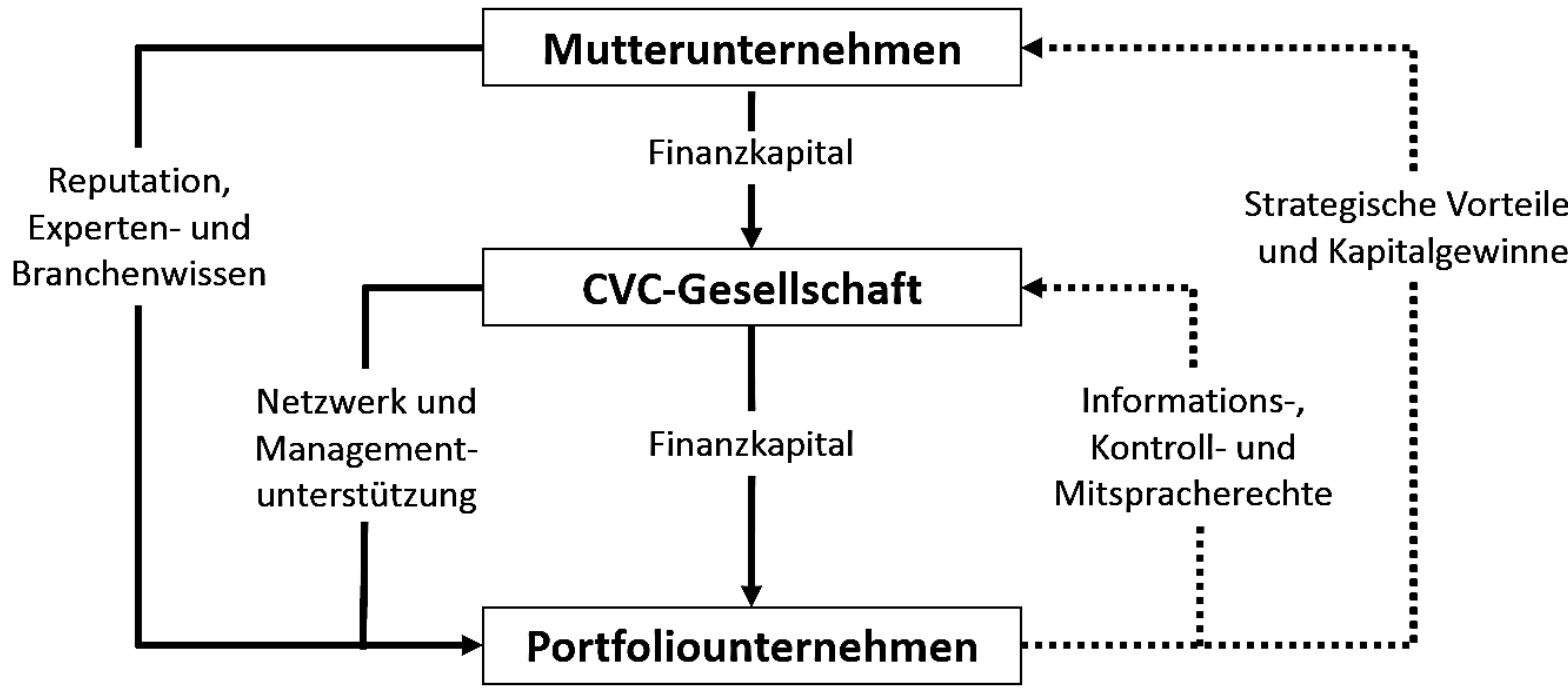

Zunächst soll die Managementunterstützung der CVC-Gesellschaften betrachtet werden, welche zwei Komponenten umfasst. Erstens kann ein Wissenstransfer in Form von Beratungsleistungen erfolgen. Häufig ist diese Art der Leistung mit einem Sitz im Aufsichts rat oder Board verbunden, wodurch das Know-how des CVC-Gesellschaft-Vertreters bei etwaigen Problemen zum Tragen kommt. ${ }^{48}$ Unter Berücksichtigung der Prinzipal-AgentTheorie zeigt sich, dass durch die Beratungsleistungen auch der CVC-Gesellschaft (Prinzipal) ein Vorteil entsteht. Demnach verfügt sie über die Option, die Tätigkeiten der Gründer (Agenten) zu beobachten. ${ }^{49}$ Dieses Monitoring nimmt aufgrund der Moral HazardProblematik eine wichtige Funktion ein. Es liegt eine Unsicherheit beim Prinzipal bezüglich der Agenten-Verhaltensmerkmale Anstrengung, Fleiß sowie Sorgfalt vor. Die Hand-

\footnotetext{
${ }^{47}$ Vgl. Schefczyk (1998), S. 41.

48 Vgl. Neubecker(2006), S. 159.

49 Vgl. Sahlman (1990), S. 508.
} 
lungen des Agenten bleiben dem Prinzipal dabei selbst ex post verborgen (Hidden Action). ${ }^{50}$ Mithilfe des Monitoring kann die CVC-Gesellschaft die Informationsasymmetrien abbauen und eine Reduktion des opportunistischen Verhaltens erreichen.

Als zweite Komponente beinhaltet die Managementunterstützung eine operative Hilfe, zu der immaterielle und materielle Leistungen gezählt werden können. Erstere umfassen beispielsweise eine Unterstützung bei der Implementierung von Unternehmensprozessen. ${ }^{51}$ Der Fokus liegt allerdings auf der materiellen Förderung, die in Form von sogenannten Overhead-Ressourcen auftritt. Eine geeignete Infrastruktur mit Büroräumen, Laboreinrichtungen sowie IT-Ausstattung sind als Teil der Förderung durch die CVC-Gesellschaften anzusehen. ${ }^{52}$

Daneben erhält das Portfoliounternehmen weitere Hilfsleistungen, was beispielsweise das bestehende Netzwerk an Kontakten der CVC-Gesellschaft einschließt. Im Bedarfsfall kann mithilfe dieser Verbindungen ein Zugang zu Vertriebskanälen sowie eine Unterstützung durch die FuE-Abteilung des Mutterunternehmens ermöglicht werden. Das Großunternehmen verfugt im Gegensatz zu vielen anderen Finanzkapitalgebern über ein spezifisches Experten- und Branchenwissen und kann den Ventures eine Beratungsleist ung zukommen lassen, welche sonst nicht allgemein verfügbar wäre. Die CVC-Gesellschaft fungiert bei der Netzwerk-Unterstützung als Intermediär und vermittelt die Kontakte zu den internen und externen Spezialisten. ${ }^{53}$

Darüber hinaus kann ebenfalls ein „Reputationstransfer“ durch das Mutterunternehmen stattfinden, welchem insbesondere aufgrund der bestehenden Informationsasymmetrie $n$ auf den Märkten eine wichtige Bedeutung zukommt. ${ }^{54}$ Durch die Zusammenarbeit mit einem bekannten Großunternehmen besteht für das Venture die Option, zusätzliche Kunden und Lieferanten zu gewinnen beziehungsweise weitere Finanzkapitalgeber von der Qualität des Portfoliounternehmens zu überzeugen. ${ }^{55}$

Die Unterstützungsphase umfasst nur den vertraglich festgelegten Zeitrahmen und endet mit Beginn der sogenannten Exit-Stufe. Dieser oft aktiv herbeigeführte Ausstieg aus dem Investment kann beispielsweise durch einen Trade Sale oder ein Initial Public Offering

\footnotetext{
50 Vgl. Schween (1996), S. 144 f.

51 Vgl. Neubecker (2006), S. 159.

52 Vgl. Haid (2004), S. 267; Neubecker (2006), S. 96.

53 Vgl. Schween (1996), S. 111; Witt (2005), S. 269.

${ }^{54}$ Vgl. Fried/Hisrich (1995), S. 104.

${ }^{55}$ Vgl. Winters/Murfin (1988), S. 211; Schween (1996), S. 111 f.
} 
erfolgen. ${ }^{56}$ Zudem kann eine vollständige Eingliederung des Ventures ins Mutterunternehmen stattfinden, um damit eine Form des Technologietransfers zu realisieren. ${ }^{57}$

\section{Fallstudie über den Main Incubator}

Der Main Incubator wurde im Oktober 2013 als hundertprozentige Tochtergesellschaft der Commerzbank in Frankfurt am Main gegründet. Der Aufbau dieser CVC-Gesellschaft kann als Reaktion des Mutterunternehmens auf einen Paradigmenwechsel im Bankensektor verstanden werden, der auf die zunehmenden Aktivitäten von sogenannten FinTechUnternehmen zurückgeht.

Bei der Bezeichnung „FinTech“ handelt es sich um eine Wortkontamination aus den Begriffen „Finanzdienstleistungen“ und „Technologien“, die eine immer weiter voranschreitende Digitalisierung des Finanzsektors beschreibt. Neben zahlreichen jungen Wirtschaftseinheiten (zum Beispiel Number26, Cringle und Paymill) können auch große, etablierte Organisationen (zum Beispiel Google, Facebook und Amazon) bei bestimmten Themen zu den FinTech-Akteuren gezählt werden. Die angebotenen Produkte sind dabei als internetbasierte Technologien $\mathrm{zu}$ charakterisieren und können als Substitute für klassische Bankprodukte angesehen werden. ${ }^{58}$

\subsection{Die Ziele des Main Incubators}

Aufgrund der beschriebenen Situation steht beim Main Incubator nicht die Steigerung der Beteiligungswerte im Vordergrund. Vielmehr ist die CVC-Gesellschaft auf die Gestaltung strategischer Vorteile ausgerichtet. In dieser Hinsicht strebt die Tochtergesellschaft an, einen Zugang zu den externen Ideen für die Commerzbank zu schaffen, welche von den jungen FinTech-Unternehmen realisiert werden. Durch die Bereitstellung von CVC soll eine Kooperation mit den jungen Wirtschaftseinheiten entstehen, die es dem Mutterunternehmen ermöglicht, sein Produktportfolio auf das neue Marktumfeld auszurichten. Als Resultat können insbesondere bestehende Firmenkunden auf digitale Neuerungen zurückgreifen, wodurch eine Stärkung der Kundenbindung erreicht werden soll.

\footnotetext{
56 Vgl. Lorenz/Seeliger (2000), S. 662.

57 Vgl. Witt (2005), S. 270.

58 Vgl. Deutsche Bank Research (2014), S. 5.
} 
Allerdings übernimmt die Commerzbank die Produkte nicht in Gänze, sondern das jeweilige FinTech-Unternehmen bleibt auch als Venture der Eigentümer. Das Großunternehmen agiert vielmehr als Intermediär und erhält dafür die Möglichkeit durch die externen Innovationen auch neue Kunden hinzuzugewinnen. Diese können Bankdienstleistungen nachfragen, die als originäre Produkte der Commerzbank anzusehen sind. In Folge dieses Cross-Selling-Effektes kann die Bank zusätzliche Erlöse generieren.

Neben der Erweiterung des Produktportfolios besteht eine zusätzliche Förderung von Neuerungen in Form eines „Window on Technology“. Es werden abseits der reinen Portfolioaktivitäten auch Marktentwicklungen durch den Main Incubator beobachtet, um aktuelle Trends zu identifizieren. In der Folge soll die Commerzbank in die Lage versetzt werden, zukünftige Kundenbedürfnisse rechtzeitig erfassen und befriedigen zu können.

\subsection{Der Markt für FinTech-Unternehmen}

Der Fokus des Main Incubators liegt auf FinTech-Unternehmen, die den Finanzierungsphasen von der Seed-Stufe bis zur First Stage angehören. Diese Eingrenzung spiegelt sich auch beim Beteiligungskapital wider, sodass Volumina bis zu geringen Millionenbeträgen bereitgestellt werden können. Zur Veranschaulichung der Zielgruppe kann das Portfolio des Main Incubators betrachtet werden, dass zurzeit aus drei Ventures besteht: ${ }^{59}$ Traxpay, Gini und OptioPay.

Im deutschen Bankensektor können für den Main Incuabtor nur wenige Wettbewerber identifiziert werden. Die Hamburger Sutorbank verfugt über eine Start-up-Plattform, die Expertenwissen und Infrastruktur bereitstellt, jedoch keine Überlassung von Beteiligungskapital vorsieht. Eine ähnliche Situation kann bei der Deutschen Bank und der Fidor-Bank erkannt werden. Die Deutsche Bank bietet jungen Unternehmen Betreuungsleistungen im Rahmen einer Kundenbeziehung an, während die Fidor-Bank ihre IT-Infrastruktur sowie ihren Namen zur Verfügung stellt. Zum CVC-Bereich sind diese Konkurrenzunternehmen nicht zu zählen. Vielmehr kann der Main Incuabtor neben CommerzVentures aktuell als einzige aktive CVC-Gesellschaft in Deutschland eingestuft

\footnotetext{
${ }^{59}$ Stand: Oktober 2015.
} 
werden, die von einer Großbank gegründet wurde. ${ }^{60}$ Darüber hinaus bezeichnet sich der Main Incubator selbst als „First Mover“ im FinTech-Bereich für Deutschland.

Vergleichbare Organisationen können jedoch in anderen europäischen Ländern identifiziert werden. Für Österreich kann die CVC-Gesellschaften BeeOne aufgeführt werden, welche von der Ersten Bank gegründet wurde. Ein weiteres Beispiel stellt die von der spanischen Banco Bilbao Vizcaya Argentaria aufgebaute Tochtergesellschaft Banco Bilbao Vizcaya Argentaria Ventures dar.

\subsection{Die Deal Flow Anbahnung des Main Incubators}

Die Auswahl von jungen Unternehmen durch den Main Incubator beschreibt einen Prozess, der aus vier Phasen besteht und mit der Deal Flow-Stufe eingeleitet wird. Die Tochtergesellschaft erhält eine Vielzahl initiativer Bewerbungen, die hauptsächlich auf dem Bekanntheitsgrad der CVC-Gesellschaft beruhen. Der Aufbau des Namens ist seit der Gründung vor allem durch Präsenz bei Veranstaltungen sowie in den Social-Media- Kanälen forciert worden.

Ergänzend wird eine aktive Vorgehensweise verfolgt. Im Rahmen der Trendanalyse für den FinTech-Markt werden gezielt junge Unternehmen angesprochen, um sie auf die CVC-Gesellschaft aufmerksam zu machen. In diesem Kontext kann auch die Initiative „Between the Towers“ gesehen werden. Sie ist vom Main Incubator gegründet worden, um ein „Ökosystem für FinTechs“ in Frankfurt am Main aufzubauen. Unter dieser Initiative ist eine Veranstaltungsreihe $\mathrm{zu}$ verstehen, bei der die gesamte FinTech-Branche in einem zeitlichen Turnus zusammenkommt und damit als gemeinsamer Treffpunkt fungiert. Bisher konnte sich eine solche zentrale Anlaufstelle in Deutschland nicht etablieren, sodass Frankfurt am Main sukzessiv zur „FinTech-Hauptstadt“ entwickelt werden soll. Für den Main Incubator, als Initiator dieses Vorhabens, besteht auch dadurch die Option, relevante FinTech-Unternehmen zu identifizieren und eine aktive Deal Flow-Anbahnung $\mathrm{zu}$ verfolgen.

\footnotetext{
${ }^{60}$ Eine Ausnahme stellt die Berliner Volksbank dar, die das Tochterunternehmen Berliner Volksbank Ventures im Oktober 2015 gegründet hat. Allerdings verfügt diese CVC-Gesellschaft über noch keine Portfoliounternehmen und wird deshalb im Rahmen dieses Artikels nicht weiter berücksichtigt. In gleicher Weise werden ein Teil der CVC-Aktivitäten durch die VC-Gesellschaft Redstone erfüllt, wodurch das Unternehmen Berliner Volksbank Ventures eher dem indirekten CVC-Bereich angehört. Stand: Oktober 2015.
} 
Zur aktiven Vorgehensweise zählen ebenfalls die Ausschreibungen auf der Homepage des Main Incubators, durch die bestimmte Themen explizit adressiert werden. Es handelt sich um Bereiche, in denen sich in Zukunft neue Märkte für das Mutterunternehmen entwickeln können. Die potentiellen Ventures sind angehalten, ihre Lösung für diese Problemgebiete einzureichen.

Insgesamt belief sich die Bewerberanzahl bei der Commerzbank-Tochtergesellschaft bis Ende 2014 auf circa 90 junge Unternehmen, wobei die Anfragen zumeist aus dem deutschsprachigen Raum kamen. Als Ursprung beziehungsweise Quelle der Bewerbungen kann neben der generellen Bekanntheit des Main Incubators sowie Kontakten zum FinTech-Bereich und anderen Investoren zudem das Commerzbank-Netzwerk aufgeführt werden. Dahingehend werden Beteiligungsmöglichkeiten auch von Mitarbeitern des Mutterunternehmens an den Main Incubator weitergeleitet.

\subsection{Das Initial Screening des Main Incubators}

Die Beteiligungswürdigkeitsprüfung der CVC-Gesellschaft kann als dreistufiges Screening verstanden werden, um die Informationsdefizite bezüglich der Bewerberqualität zu reduzieren. Abbildung 3 ist als schematische Darstellung dieses Prozesses anzusehen, welcher zumeist mit der Einreichung des Businessplanes beginnt:

\section{Abbildung 3: Auswahlprozess des Main Incubators.}

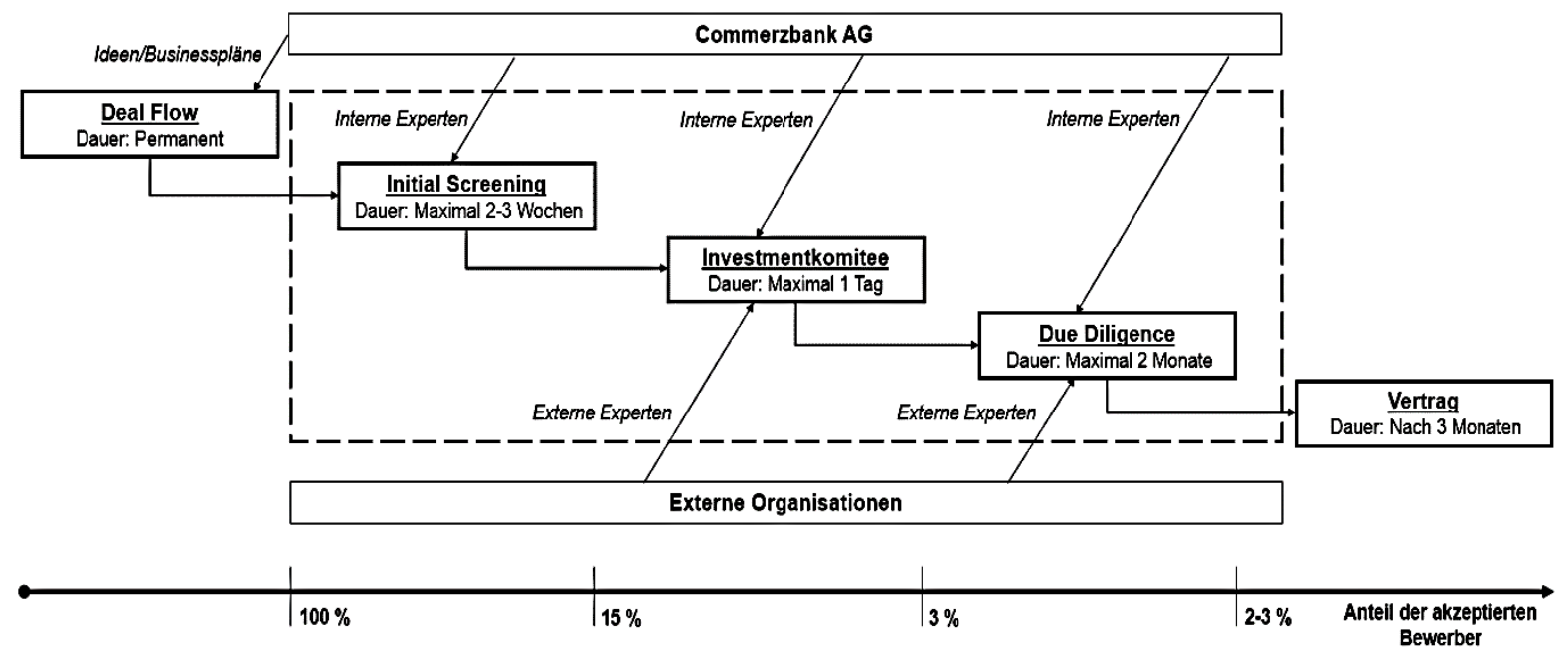

Während des Initial Screening werden die Beteiligungsmöglichkeiten hauptsächlich von den Investmentmanagern des Main Incubators analysiert, welche ein breites Fachwissen abbilden. Mithilfe unterschiedlicher Perspektiven soll die Möglichkeit bestehen, die verschiedenen Auswahlkriterien zu kontrollieren. Die wichtigsten Merkmale sind dabei: 
- Businessplan: Der Businessplan als einleitender Impuls muss noch nicht vollständig sein. Allerdings sollten die wesentlichen Punkte, Geschäftsmodell, Wettbewerber, Zielgruppe beziehungsweise möglicher Markt, finanzielle Faktoren sowie Teamzusammenstellung, aufgeführt werden. Die Gestaltung des Businessplans gibt zudem wichtige Auskünfte darüber, ob die Gründer überhaupt die Fähigkeiten besitzen, ein Geschäftsmodell mit fundierten Annahmen zu entwickeln.

- Produktidee: Im Vergleich zu den bisher vorhandenen Produkten soll die Entwicklung eine absolute Neuheit darstellen und der situativen Relevanz der Kunden entsprechen. Bei dieser Beurteilung erfolgt eine Unterstützung durch das Mutterunternehmen beziehungsweise den jeweiligen internen Produkt-Spezialisten, sodass auch das inhärente Wissen der Commerzbank eingesetzt werden kann. Insgesamt nehmen die Mutterunternehmen-Experten aber nur eine beratende Funktion ein.

- Gründerteam: Während des Initial Screening finden mehrere persönliche Treffen und Telefongespräche statt, um die handelnden Personen einzuschätzen. Dabei treten Faktoren wie die Branchenkenntnis, die Teamzusammenstellung und der berufliche Werdegang in den Vordergrund. Es werden vor allem Gründer präferiert, die schon mehrere Jahre im Finanzbereich gearbeitet haben und Gründungserfahrung aufweisen.

- Regulatorik: Ebenfalls sind die gesetzlichen Voraussetzungen für den Bankenbereich von Belang, welche häufig von den FinTech-Unternehmen unterschätzt werden. Hierbei gilt es zu überprüfen, ob mögliche Lizenzbestimmungen existieren und ob diesen von den Bewerbern entsprochen wird.

Nach einem positiven Initial Screening (inklusive Sounding in der Commerzbank) wird ein sogenanntes Proposal erstellt, welches die wichtigsten Informationen über das FinTech-Unternehmen beinhaltet und die Grundlage der nächsten Überprüfungsstufe darstellt. Überdies soll schon zu diesem Zeitpunkt geklärt sein, wie hoch die Bewertung des jungen Unternehmens ist und welches Investitionsvolumen der Main Incubator bereitstellen würde. 
Als zweite Stufe sieht der Main Incubator den Einsatz eines Investmentkomitees vor, das einmal im Quartal zusammenkommt. Die Mitglieder dieses Ausschusses bestehen vorwiegend aus externen Experten und bilden ebenfalls eine breite Expertise ab. Demnach sind ein Rechtsanwalt, ein Trendforscher, ein Chief Financial Officer von einem Softwareunternehmen, ein kaufmännischen Leiter sowie ein Marketingspezialist in diesem Komitee vertreten. Ebenso umfasst der Ausschuss einen Vorstand und einen Bereichsvorstand der Commerzbank. Neben der damit verbundenen Erfahrung im Bankensektor soll dadurch die spätere Integration der FinTech-Produkte in das Mutterunternehmen erleichtert werden.

Diese Überprüfungsstufe basiert auf drei Komponenten: Als Basis kann das bereits nach dem Initial Screening angefertigte Proposal angesehen werden. Anschließend müssen die ausgewählten FinTech-Unternehmen ihr Konzept beziehungsweise ihre Geschäftsidee vor dem Investmentkomitee präsentieren, wodurch dieses einen eigenen Eindruck von dem jungen Unternehmen erhält. Abschließend findet eine Frage-Antwort-Runde statt, um weiterhin bestehende Unklarheiten anzusprechen.

\subsection{Die Due Diligence des Main Incubators}

Eine positive Bewertung des Investmentkomitees vorausgesetzt, beginnt der letzte $\mathrm{Ab}$ schnitt des Screening, welcher die eigentliche Due Diligence umfasst. Soweit es die Entwicklungsphase des FinTech-Unternehmens zulässt, erfolgt eine intensive Überprüfung der Bewerber. Hierbei werden auch solche Faktoren analysiert, die das junge Unternehmen noch nicht beim Initial Screening offengelegt hat. Nur in Ausnahmefällen kann es in dieser Phase noch zur Ablehnung eines Bewerbers kommen. Parallel zu dieser Analyse finden ebenfalls die Verhandlungen für den Beteiligungsvertrag statt, welcher zwischen der CVC-Gesellschaft und dem FinTech-Unternehmen geschlossen wird. Bei der Due Diligence kommen, wie in der vorherigen Phase, externe und interne Experten zum Einsatz. Erstere überprüfen vor allem die IT-Komponenten, sowie die Patentrechte. Bedingt durch den technologischen Charakter der FinTech-Unternehmen wird dieser Schritt bei jedem potentiellen Venture durchgeführt.

Die Beteiligungswürdigkeitsprüfung nimmt längstenfalls einen Zeitrahmen von drei Monaten ein. Insgesamt betrachtet, liegt der Schwerpunkt des gesamten Auswahlprozesses 
auf dem Initial Screening, da schon hier viele Inhalte einer Due Diligence realisiert werden und eine umfassende Selektion stattfindet. Ende des Jahres 2014 haben circa drei Prozent aller Bewerbungen nach der Beteiligungswürdigkeitsprüfung eine positive Votierung erhalten.

\subsection{Die Beteiligungsverträge mit den FinTech-Unternehmen}

In den Beteiligungs verträgen können Meilensteine aber auch Informationsrechte festgeschrieben werden. Generell soll eine weitreichende Autonomie der Ventures hinsichtlich der Kontroll- und Mitspracherechte erhalten bleiben, damit die Eigeninitiative der Gründer nicht zu stark eingeschränkt wird. Zudem geht die CVC-Gesellschaft nur Minderheitsbeteiligungen ein, sodass für die Portfoliounternehmen weiterhin ein Anreiz besteht, ein umfangreiches Engagement zu zeigen.

\subsection{Die Unterstützungsleistungen der Commerzbank}

Für die vom Main Incubator ins Portfolio aufgenommen Ventures beginnt nach dem Vertragsabschluss die operative Zusammenarbeit. Während dieses Abschnittes erhalten die FinTech-Unternehmen neben dem Beteiligungskapital verschiedene nicht-finanzielle Leistungen, um die Zeit bis zum Markteintritt (Time-to-Market) zu reduzieren. Hierbei kann eine Differenzierung zwischen dem Beitrag des Mutterunternehmens und der Tochtergesellschaft stattfinden. Die Commerzbank tritt vor allem bei folgenden Punkten in den Vordergrund:

- Beratungsleistung: Bei möglichen Neu- oder Weiterentwicklungen sowie dem Vertrieb der FinTech-Produkte stellt die Commerzbank das entsprechende Expertenwissen bereit. Zudem übernehmen die Spezialisten eine wichtige Beratungsfunktion hinsichtlich der damit verbundenen rechtlichen Bedingungen.

- Operative Unterstützung: In gleicher Weise kann eine operative Unterstützung erfolgen, was gleichermaßen den Einsatz von Experten mit einschließt. Es handelt sich hierbei um IT-Spezialisten, welche für die Eingliederung der Venture-Produkte in das Commerzbank-System zuständig sind. Eine vollständige Übernahme operativer Aufgaben, wie zum Beispiel bei der Buchhaltung oder der Organisation des Zahlungsverkehrs, ist jedoch nicht vorgesehen. 
- Reputation: Daneben kann die Reputation der Commerzbank als eine indirekte Hilfsleistung bezeichnet werden. Der Name des Main Incubators wurde zwar bewusst nicht an die Bezeichnung des Mutterunternehmens angelehnt, um eine von der Commerzbank losgelöste Denkweise zu repräsentieren. Dennoch können eine Vielzahl an Bewerbungen bei der Tochtergesellschaft auf das eigentliche Mutterunternehmen zurückgeführt werden. Das Commerzbank-Renommee ist vor allem für die Gewinnung von neuen Investoren und Kunden ein förderlicher Faktor.

- Kundenzugang: Durch die Aufnahme der FinTech-Produkte in das Produktportfolio der Commerzbank steht das gesamte Kundenpotenzial für die Ventures zur Verfügung. Bestehende Markteintrittsbarrieren können dadurch für die jungen Unternehmen reduziert werden, welche sich aufgrund fehlender positiver Referenzen (Track-Record) ergeben. Insgesamt betreut das Großunternehmen rund 15 Millionen Privat- sowie eine Millionen Geschäfts- und Firmenkunden.

3.9 Die Unterstützungsleistungen des Main Incubators

Der Main Incubator übernimmt in gleicher Weise wie das Mutterunternehmen verschiedene nicht-finanzielle Leistungen. Hierbei lassen sich folgende Aufgabenfelder unterscheiden:

- Vermittlungsfunktion: Die Tochtergesellschaft ist primär für die Weiterentwicklung des Portfoliounternehmens verantwortlich. Dahingehend kann eine Unterstützung bei der Durchführung neuer Finanzierungsrunden oder der Suche nach zusätzlichen Investoren erfolgen. Durch das bestehende Netzwerk an Kontakten übernimmt der Main Incubator eine Vermittlungsfunktion. Diese Intermediationsaufgabe erfuillt die CVC-Gesellschaft auch für die Anbahnung der CommerzbankFörderung. Mithilfe einer Schnittstelle zum Mutterunternehmen, werden relevante Aufgaben an die Bank kommuniziert. Ergänzend kommen hierbei die Mitarbeiterkontakte der Tochtergesellschaft in das Großunternehmen zum Tragen.

- Beratungsleistung: Überdies stellt der Main Incubator spezielles Branchen- beziehungsweise Fachwissen zur Verfügung, was insbesondere eine Beratung zu Trends und Entwicklungen im Finanzbereich impliziert. In diesem Kontext ist zudem die Stellung von Mitgliedern des Aufsichtsrates oder des Boards zu sehen. Neben dem Main Incubator übernimmt allerdings auch die Commerzbank eine solche Beratungsfunktion, was beispielsweise bei Traxpay der Fall ist. Es wird 
eine situative Entscheidung getroffen, welche Organisation den Ausschuss vertreter stellt oder ob sogar auf den Aufbau eines solchen Gremiums verzichtet wird.

- Operative Unterstützung: Gleichermaßen wird eine operative Unterstützung durch den Main Incubator übernommen. Schwerpunktmäßig erfolgt eine Bereitstellung von Büroräumen und IT-Infrastruktur. Allerdings handelt es sich nur um eine temporäre Leistung, welche insbesondere für Portfoliounternehmen in der Seed- und Start-up-Phase vorgesehen ist, die noch aus kleinen Gründerteams bestehen. ${ }^{61}$

\subsection{Dauer der Unterstiitzungsphase}

Der Unterstïtzungsabschnitt umfasst zunächst einen Zeitabschnitt von sechs Monaten. Im Anschluss an diesen Zeitraum erfolgt eine erneute Präsentation vor dem Investmentkomitee, welches die Fortschritte bewertet und über die weitere Zusammenarbeit abstimmt. Hierbei kann auch eine Erhöhung des Finanzkapitals für das Portfoliounternehmen beschlossen werden, falls entsprechende monetäre Defizite bestehen sollten. Bei einer neuerlichen positiven Votierung hat das Venture zusätzliche sechs Monate Zeit, sein Produkt weiterzuentwickeln. Nach Ablauf dieser Frist sollte jedoch eine Markteinführung des Produktes möglich sein. Einen positiven Verlauf vorausgesetzt kann eine langfristige Kooperation zwischen Portfoliounternehmen und Main Incubator entstehen.

\section{Analyse der Ergebnisse}

Der Aufbau und das Vorgehen des Main Incubators entsprechen weitestgehend den bestehenden Annahmen zum CVC. Allerdings können zwei markante Ergebnisse festgehalten werden, welche auf die spezifische Branchenzugehörigkeit der Tochtergesellschaft zurückzuführen sind:

- Zunächst stellt die Berücksichtigung von regulatorischen Voraussetzungen beim Initial Screening einen wesentlichen Unterschied dar. Der Bankenbereich unterliegt umfassenderen gesetzlichen Bestimmungen als viele andere Wirtschaftsbereiche, was sich auch bei der Auswahl von Portfoliounternehmen widerspiegelt. Vor einer Beteiligung wird überprüft, inwieweit den gesetzlichen Vorgaben ent-

\footnotetext{
${ }^{61}$ Aufgrund dieser Unterstützungs leistung kann der Main Incubator auch als sogenannter Corporate Incubator bezeichnet und zur Gruppe der „Inkubatoren“ gezählt werden.
} 
sprochen wird und ob bereits notwenige Lizenzen, wie zum Beispiel Banklizenzen oder E-Money-Lizenzen, vorliegen. Banklizenzen versetzen Wirtschaftseinheiten in die Lage klassische Bank- beziehungsweise Finanzdienstleistungsgeschäfte zu betreiben. ${ }^{62}$ Die zweite Lizenzart berechtigt wiederum Unternehmen dazu, sogenannte E-Geld-Geschäfte für ihre Kunden anzubieten. ${ }^{63}$

- In gleicher Weise gilt es, die regulatorischen Bedingungen bezüglich der Unterstützungsleistungen aufzuführen. Nach den bisherigen Erfahrungen des Main Incubators fehlt es den FinTech-Unternehmen häufig am entsprechenden Wissen und an den Voraussetzungen, um den gesetzlichen Anforderungen für den Bankenbereich nachzukommen. Dies führt dazu, dass entweder vollends auf Lizenzen verzichtet wird oder sachkundige Rechtsanwälte konsultiert werden müssen, wodurch hohe Transaktionskosten entstehen. Folglich ermöglicht die CVC-Gesellschaft seinen Portfoliounternehmen, auf entsprechende Beratungsleistungen zurückzugreifen. Dadurch sollen bestehende Markteintrittsbarrieren beziehungsweise Wettbewerbsnachteile für die Ventures reduziert werden. Aber auch für die Wahrnehmung in der Öffentlichkeit haben die regulatorischen Vorgaben eine wichtige Bedeutung. Mögliche Verstöße durch die Portfoliounternehmen des Main Incubators können Auswirkungen auf die Außendarstellung der Commerzbank haben und zu einer negativen Berichterstattung in der Presse führen.

Unter Berücksichtigung der Prinzipal-Agent-Theorie können zwei zusätzliche Resultate für diese Fallstudie festgehalten werden:

- Abweichend von den bestehenden Annahmen übernimmt nicht ausschließlich der Main Incubator (Prinzipal) Posten im Aufsichtsrat beziehungsweise Board bei den Portfoliounternehmen. Vielmehr erfolgt eine situative Entscheidung, ob Mitarbeiter des Mutterunternehmens oder der Tochtergesellschaft eine Funktion im Ausschuss der jungen Unternehmen (Agent) wahrnehmen. Die Einzelfallbetrachtung des Main Incubators verdeutlicht diesen Aspekt. Bei jedem Venture werden individuelle Überlegungen vorgenommen, wodurch eine geeignete Beratungsleistung sowie Kontrolle der Ventures erfolgen kann. Durch dieses spezifische Monitoring besteht die Option, dass gegebene Informationsasymmetrien umfassender abgebaut werden können, was insbesondere in Hinblick auf die Moral Hazard-Problematik bedeutsam ist.

${ }^{62} \mathrm{Vgl}$. Kreditwesengesetz $\$ 32 \mathrm{KWG}$.

${ }^{63} \mathrm{Vgl}$. Zahlungsdienstaufsichtsgesetz § $32 \mathrm{ZAG}$. 
- Die vorliegende Fallstudie zeigt, dass interne Experten des Mutterunternehmens sowie externe Spezialisten eine wichtige Funktion für das gesamte Screening übernehmen. Um zusätzliches Know-how für die Qualitätsprüfung der Bewerber bereitzustellen, empfiehlt es sich für CVC-Gesellschaften, auf den Einsatz solcher Experten zurückzugreifen. Bei möglichen Substituten von originären Bankprodukten kann allerdings die Gefahr bestehen, dass für die internen Spezialisten (Agenten) Interessenskonflikte auftreten, die eine objektive Bewerberbeurteilung erschweren. Eine Lösung dieser Problematik kann anhand des Main Incubators erkannt werden. Der Einsatz von internen Experten wird bei der Tochtergesellschaft (Prinzipal) auf eine beratende Funktion beschränkt. Mögliche Interessenskonflikte sollen dadurch vermieden und gleichzeitig das Fachwissen der Commerzbank-Mitarbeiter nutzbar gemacht werden.

\section{Fazit}

In dem vorliegenden Artikel erfolgte eine theoretische und empirische Untersuchung des bisher wenig erforschten CVC im Rahmen einer Fallstudie. Für die empirische Grundlage wurde dabei ein neuartiger Fall ausgewählt. In Form des Unternehmens Main Incubator konnte auf eine CVC-Gesellschaft aus dem Bankenbereich zurückgegriffen werden. Es handelt sich um eine Branche, welche in der Literatur sonst nicht zum CVC-Bereich gezählt wird. Die umfassenderen gesetzlichen Bestimmungen, die für Banken gelten, stellen dabei einen wesentlichen Unterschied zu den CVC-Aktivitäten in anderen Wirtschaftsbereichen dar, was sich sowohl in den Auswahlkriterien als auch in den Unterstützungsle istungen widerspiegelt.

Für weiterfürende Forschungen bietet es sich an, zusätzliche Untersuchungsobjekte hinzuzuziehen und in Form einer Mehrfallstudie zu analysieren. Mithilfe einer solch vergle ichenden Forschungsarbeit kann aufgezeigt werden, ob auftretende Befunde sich gegebenenfalls bei anderen Fällen wiederholen. ${ }^{64}$ Allerdings existieren aktuell nur der Main Incubator und CommerzVentures als aktive CVC-Gesellschaften aus dem Bankensektor in Deutschland.

In diesem Zusammenhang kann allerdings eine Analogie $\mathrm{zu}$ einem anderen Wirtschaftsbereich entstehen. Die Medien- und Verlagsbranche steht seit geraumer Zeit unter dem

\footnotetext{
${ }^{64}$ Vgl. Eisenhardt/Graebner(2007), S. 27.
} 
Druck, sich wandelnder Strukturen aufgrund von neuen internetbasierten Technologien. ${ }^{65}$ Bei den zurzeit in Deutschland vorhandenen CVC-Gesellschaften sind eine Vielzahl an Tochtergesellschaften von Großunternehmen (zum Beispiel Bertelsmann, Verlagsgruppe Handelsblatt und M. DuMont Schauberg Mediengruppe) aus dieser Branche gegründet worden. Eine solche Entwicklung kann prinzipiell auch im Bankenbereich eintreten. Aufgrund des Innovationsdrucks durch die FinTech-Unternehmen ist es möglich, dass weitere Finanzinstitute ein ähnliches Vorgehen anstreben, wodurch eine größere Fallanzahl entstehen würde. Diese wäre ebenfalls (zumindest vorläufig) aus Sicht der FinTech-Unternehmen eine erstrebenswerte Situation. Aufgrund der regulatorischen Rahmenbedingungen sind viele FinTech-Unternehmen derzeit ohnehin darauf angewiesen, mit lizenzierten Banken zusammenarbeiten, um ihre Produkte überhaupt anbieten zu dürfen. In dieser Hinsicht könnten entsprechende CVC-Programme von Banken eine adäquate Unterstützung für die Entwicklung und Marktakzeptanz der FinTech-Unternehmen darstellen.

\section{Literatur}

Albach, Horst (1994), Culture and Technical Innovation: A Cross-Cultural Analysis and Policy Recommendations, Berlin.

Amit, Raphael/Glosten, Lawrence/Muller, Eitan (1990), Does Venture Capital-Foster the Most Promising Entrepreneurial Firms?, in: California Management Review, Vol. 10, S. 102-111.

Chesbrough, Henry William (2006), Open Innovation: The New Imperative for Creating and Profiting from Technology, Boston.

Chesbrough, Henry William/Socolof, Stephen (2000), Creating New Ventures from Bell Labs Technologies, in: Research Technology Management, Vol. 43, S. 13-17.

Christensen, Clayton (1997), The Innovator's Dilemma: Warum etablierte Unternehmen den Wettbewerb um bahnbrechende Innovationen verlieren, aus dem Amerikanischen übersetzt und überarbeitet von Matzler, Kurt/Eichen, Stephan von den, München.

Deutsche Bank Research (2014), Fintech - The Digital (R)evolution in the Financial Sector.

Eisenhardt, Kathleen/Graebner, Melissa (2007), Theory Building from Cases: Opportunities and Challenges, in: Academy of Management Journal, Vol. 50, S. 25-32.

\footnotetext{
65 Vgl. Deutsche Bank Research (2014), S. 5.
} 
Fendel, Andreas (1987), Investmententscheidungsprozesse in Venture-Capital-Unternehmungen: Darstellung und Möglichkeiten der instrumentellen Unterstützung, Köln.

Fried, Vance/Hisrich, Robert (1995), The Venture Capitalist: A Relationship Investor, in: California Management Review, Vol. 37, S. 101-113.

Gompers, Paul/Lerner, Josh (2000), The Determinants of Corporate Venture Capital Success: Organizational Structure, Incentives, and Complementarities, in Morck, Randall (Hrsg.), Concentrated Corporate Ownership, Chicago, S. 17-50.

Greenhalgh, Christine/Rogers, Mark (2010), Innovation, Intellectual Property, and Economic Growth, Princeton.

Gumpert, David (1994), Creating a Successful Business Plan, in: Bygrave, William

(Hrsg.), The Portable MBA in Entrepreneurship, New York, S. 120-147.

Haid, Dirk (2004), Corporate Entrepreneurship im strategischen Management, Wiesbaden.

Hardenberg, Colin von (1989), Die Bereitstellung von Venture Capital durch Großunternehmen: Ein Mittel zur Sicherung und Aufdeckung ihrer Entwicklungsmöglichkeiten, Göttingen.

Heister, Peter (2010), Finanzierung von Social Entrepreneurship durch Venture Philanthropy und Social Venture Capital: Auswahlprozess und -kriterien der Finanzintermediäre, Wiesbaden.

Kollmann, Tobias (2003), Unternehmensbeteiligung im Electronic Business, in: Wirtschaftswissenschaftliches Studium, 32. Jg., S. 322-327.

Kreysel, Michael (2006), Venture Capital als Instrument der Innovationsfinanzierung von Großunternehmen: Eine theoretische und empirische Analyse, 1. Aufl., Dresden.

Kreditwesengesetz $\$ 32$ Abs. 1 KWG in der Fassung der Bekanntmachung vom 9. Sep tember 1998 (Bundesgesetzblatt I S. 2776), das zuletzt durch Artikel 1 des Gesetzes vom 15. Juli 2014 (Bundesgesetzblatt I S. 934) geändert worden ist.

Lerner, Josh (2010), Innovation, Entrepreneurship and Financial Market Cycles, OECD Science, Technology and Industry Working Papers 2010/03.

Lerner, Josh/Tåg, Joacim (2013), Institutions and Venture Capital, in: Industrial and Corporate Change, 22. Jg., S. 153-182.

Lorenz, Matthias/Seeliger, Carsten (2000), Corporate Venture Capital: Konzerne realisieren nicht nur hohe Renditen, sondern erschließen auch Innovationspotenzial, in: Finanz-Betrieb, 2. Jg., S. 658-662.

McNally, Kevin (1997), Corporate Venture Capital: Bridging the Equity Gap in the Small Business Sector, 1. Aufl., London. 
Meier, Heinz Michael/Rasmussen-Bonne, Hans-Eric (2000), Ablauf der Beteiligungsverhandlungen bis hin zum Beteiligungsvertrag, in: Weitnauer, Wolfgang (Hrsg.), Handbuch Venture Capital: Von der Innovation zum Börsengang, München, S. 261-289.

Neubecker, Jochen (2006), Finanzierung durch Corporate Venture Capital und Venture Capital: Empirische Untersuchung zum Value Added junger, innovativer Unternehmen in Deutschland, 1. Aufl., Wiesbaden.

Poser, Timo (2003), The Impact of Corporate Venture Capital: Potentials of Competitive Advantages for the Investing Company, 1. Aufl, Wiesbaden.

Sahlman, William (1990), The Structure and Governance of Venture-Capital Organizations, in: Journal of Financial Economics, Vol. 27, S. 473-521.

Schefczyk, Michael (1998), Erfolgsstrategien deutscher Venture Capital-Gesellschaften, Stuttgart.

Schröder, Christoph (1992), Strategien und Management von Beteiligungsgesellschaften: Ein Einblick in Organisationsstrukturen und Entscheidungsprozesse von institutionellen Eigenkapitalinvestoren, Baden-Baden.

Schultz, Christian (2011), Die Finanzierung technologieorientierter Unternehmen in Deutschland, Wiesbaden.

Schween, Karsten (1996), Corporate Venture Capital: Risikokapitalfinanzierung deutscher Industrieunternehmen, Wiesbaden.

Siegel, Robin/Siegel, Eric/MacMillan, Ian (1988), Corporate Venture Capitalists: Autonomy, Obstacles and Performance, in: Journal of Business Venturing, Vol. 3, S. 233-247.

Stringer, Robert (2000), How to Manage Radical Innovation, in: California Management Review, Vol. 42, S. 77-80.

Sykes, Hollister (1990), Corporate Venture Capital: Strategies for Success, in: Journal of Business Venturing, Vol. 5, S. 37-47.

Utterback, James (1994), Mastering the Dynamics of Innovation: How Companies Can Seize Opportunities in the Face of Technological Change, Boston.

Winters, Terry/Murfin, Donald (1988), Venture Capital Investing for Corporate Development Objectives, in: Journal of Business Venturing, Vol. 3, S. 207-222.

Witt, Peter (2005), Corporate Venture Capital, in: Börner, Christoph/Grichnik Dietmar, Entrepreneurial Finance: Kompendium der Gründungs- und Wachstumsfinanzierung, Heidelberg.

Zahlungsdienstaufsichtsgesetz § 32 ZAG vom 25. Juni 2009 (BGBl. I S. 1506), das zuletzt 
durch Artikel 16 des Gesetzes vom 15. Juli 2014 (BGBl. I S. 934) geändert worden ist.

Zemke, Ingo (1995), Die Unternehmensverfassung von Beteiligungskapitalgesellschaften, Wiesbaden.

\section{Corporate Venture Capital in the Banking Sector: A Case Study}

\section{Summary}

Digitization of society has a strong effect on the banking sector. This circumstance is shown by the increasing success of young FinTech firms and the pressure to innovate for traditional banks. Therefore Commerzbank AG found an own corporate venture Capital firm (CVC firm), the Main Incubator GmbH, in March 2014. This CVC firm supports its parent company to cooperate with FinTech firms and to implement synergy potential for the own core business. In the research literature CVC activities are primarily assigned to the industrial and service sectors and not to the banking sector. Due to this fact Main Incubator is an unusual case, which is analyzed in a case study. The aim of this project is to show specific characteristics of a CVC firm of the banking sector. 